\title{
Spectral function and conductivity in the normal state of the cuprates: a spin fluctuation study.
}

\author{
R. Haslinger, Andrey V. Chubukov, and Ar. Abanov \\ 1 Department of Physics, University of Wisconsin, Madison, WI 53706
}

(October 24, 2018)

\begin{abstract}
We study the spectral function $A_{k}(\omega)$ and the optical conductivity $\sigma_{1}(\omega)$ for a system of fermions interacting with their collective spin fluctuations (the spin-fermion model), and apply the results to optimally doped cuprates in the normal state. We show that there is no qualitative distinction between hot and cold regions in the Brillouin zone - in both cases, there exists a wide range of frequencies where the width of the peak in $A_{k}(\omega)$ scales linearly with $\omega$. We demonstrate that $\sigma_{1}(\omega)$ is inversely linear in $\omega$ up to very high frequencies. We argue that these results agree quantitatively with the photoemission and optical data for Bi2212.

PACS numbers:71.10.Ca,74.20.Fg,74.25.-q
\end{abstract}

The observed discrepancies between the normal state properties of the cuprates and the predictions of Fermi liquid theory continue to attract a lot of attention from the condensed-matter community. According to a Fermi liquid theory, the behavior of all fermionic systems at sufficiently low energies is universal and is governed by the fact that the quasiparticle damping near the Fermi surface scales as $\omega^{2}$ or $T^{2}$ (whichever is larger). This should give rise to the $\omega^{2}\left(T^{2}\right)$ behavior of the width of the photoemission peak, to $1 / \omega^{2}$ behavior of the optical conductivity $\sigma_{1}(\omega)$ at $T=0$, and to the $T^{2}$ behavior of the resistivity $\rho(T)$.

Traces of Fermi liquid behavior have been observed in strongly overdoped cuprates. For smaller dopings, however, the deviations from the Fermi liquid behavior become substantial, and even at optimal doping, the system behavior in all experimentally accessable frequency ranges is qualitatively different from that in a Fermi liquid [1]. Amazingly enough, this non-Fermi liquid behavior can, to good accuracy, be described by simple linear functions of frequency and temperature. In particular, the width of the ARPES peak scales with $\omega$ for a wide range of frequencies, the conductivity is inversely proportional to $\omega$, and the resistivity is linear in $T$. To which extent this behavior survives in underdoped cuprates is unclear as below optimal doping, the normal state behavior is masked by the development of the pseudogap.

There are currently three qualitatively different phenomenological scenarios for the observed linearities at optimal doping. The first is a marginal Fermi liquid scenario [2]. It assumes that near optimal doping, there exists a quantum critical point of unknown origin, at which $\Sigma_{k}^{\prime \prime}(\omega)$ is linear in $\omega$ and independent of $k$. The linearity of the resistivity and inverse optical conductivity then follow from a conventional Drude theory.

The second is a cold spot scenario [3]. It assumes that the quasiparticle lifetime is very anisotropic along the Fermi surface and preserves a Fermi liquid form only in a narrow range near the zone diagonal where the quasiparticle lifetime is the longest. The width of this range is assumed to scale with $\omega$ (or $T$ ). Elementary manipulations show that the Fermi liquid region then yields the linear behavior of resistivity and inverse conductivity.

The third is a magnetic hot spot scenario which relates the linear behavior of the resistivity with the nonFermi liquid behavior due to strong spin-fluctuation scattering. This scenario is questionable on general grounds as the computation of conductivity implies averaging of the quasiparticle lifetime over the Fermi surface [4], but still may be applicable by numerical reasons.

In the present communication, we adopt a nonphenomenological approach and compute the ARPES lineshape, optical conductivity and resistivity for the spin-fermion model which describes fermions interacting with their own collective spin degrees of freedom. Two of us argued in a series of recent publications [5] that the strong coupling limit of this model captures the physics of the cuprates. We will use a previously obtained expression for the fermionic self energy $\Sigma$ as an input, and compute the photoemission intensity for various $k$, optical conductivity and resistivity.

The results we obtain partly agree and partly disagree with each of the three phenomenological scenarious above. In agreement with the marginal Fermi liquid picture, we found that $\Sigma^{\prime \prime}(\omega)$ is linear in $\omega$ and in $T$ for a wide range of frequencies everywhere on the Fermi surface. This linearity in $\Sigma^{\prime \prime}$ causes the linear behavior of both the optical conductivity and the resistivity. In contradiction with the marginal Fermi liquid scenario the linear behavior of $\Sigma^{\prime \prime}(\omega)$ in the spin-fermion model is not associated with a closeness to an unknown phase transition at optimal doping. Rather, it emerges as an intermediate asymptotics in the crossover regime between the two physically motivated limits: a Fermi liquid at the lowest frequencies, where $\Sigma^{\prime \prime} \propto \omega^{2}$ and a high frequency limit where the system is in the magnetic quantum-critical regime, and $\Sigma^{\prime \prime} \propto \sqrt{\omega}[5]$. The crossover between the two limits is governed by a single parameter - a momentum dependent typical spin relaxation frequency $\omega_{s f}(k)$. We find, however, that the crossover region is strikingly 
wide and covers the whole frequency range probed in the experiments.

In agreement with the cold spot scenario, we found that the amplitude of the scattering rate is anisotropic over the Fermi surface, and is smallest near the zone diagonals. Acoordingly, (i) the slope of $\Sigma(\omega)$ is the smallest and (ii) the crossover frequency $\omega_{s f}(k)$ is the largest for $k=k_{\text {diag }}$. However, we found that $\omega_{s f}\left(k_{\text {diag }}\right)$ is very low, $(\leq 50 \mathrm{meV}$ ), such that for $\omega$ and/or $\pi T$ probed in ARPES and conductivity experiments, the system is outside the Fermi liquid regime even for $k=k_{\text {diag }}$.

Finally, in agreement with the hot spot theory, we find that the damping rate is indeed the strongest near the hot spots, and that there is a substantial, although not dominant, contribution to the conductivity from the hot regions.

We now turn to the calculations. The fermionic selfenergy in the spin-fermion model has been obtained before [5] and is the starting point for our studies in this paper. We have

$$
\Sigma_{k}\left(\omega_{m}\right)=i \pi T \lambda \sum_{n} \frac{\operatorname{sign} \omega_{n}}{\sqrt{1+\frac{\left|\omega_{m}-\omega_{n}\right|}{\omega_{s f}}+(\tilde{k} \xi)^{2}}} .
$$

where $\omega_{m}$ and $\omega_{n}$ are fermionic Matsubara frequencies. Here $\lambda \sim \xi$ is the dimensionless effective coupling, $\tilde{k}=\left|\mathbf{k}-\mathbf{k}_{h s}\right|$ is the momentum deviation from a hot spot along the Fermi surface, and $\omega_{s f}=$ $\left(3 \sin \phi_{0} /(16 \pi)\right) v_{F} \xi^{-1} / \lambda$, where $v_{F}$ is the Fermi velocity, $\phi_{0}$ is the angle between the Fermi velocities at $k$ and $k+Q$, and $\xi-$ is the magnetic correlation length.

The physical meaning of $\omega_{s f}$ can be understood by analyzing Eq. (何) at $T=0$ and $k=k_{h s}$. In this limit, the frequency summation and transformation to a real axis can be performed exactly and yields $\Sigma_{k_{h s}}(\omega)=2 \lambda \omega /(1+$ $\left.\sqrt{1-i|\omega| / \omega_{s f}}\right)$. A simple manipulation then shows that $\omega_{s f}$ is a crossover scale between a Fermi liquid behavior at $\omega \ll \omega_{s f}$ where $\Sigma_{k_{h s}}(\omega) \approx \lambda\left(\omega+i \omega|\omega| /\left(4 \omega_{s f}\right)\right)$, and the quantum-critical $(\xi=\infty)$ behavior at $\omega \gg \omega_{s f}$ where $\Sigma_{k_{h s}}(\omega) \approx i \operatorname{sign} \omega(|\omega| \bar{\omega})^{1 / 2}$, and $\bar{\omega}=4 \lambda^{2} \omega_{s f}$ is independent on $\xi$.

In Fig. 11a,b we present plots of $\Sigma_{k_{h s}}(\omega, T)$. We computed $\Sigma^{\prime \prime}(\omega, T)$ by using the spectral representation for (11), and then used a Kramers-Kronig transform to obtain $\Sigma^{\prime}(\omega, T)$. We see that at a given $T, \Sigma^{\prime \prime}(\omega)$ is linear in $\omega$ at intermediate frequencies. At $T=0$, the linear regime extends between $0.5 \omega_{s f}$ and $8 \omega_{s f}$. This linear behavior has been observed in numerical studies [6]. In Fig.17c we see that $\Sigma_{k_{h s}}^{\prime \prime}(T)$ at fixed, small $\omega$ is linear in $T$ above $100 \mathrm{~K}$. Theoretically, the linearity in $T$ can be understood as coming primarily from the scattering by thermal, classical spin fluctuations (i.e., from the $n=m$ term in (11)). In Fig. Id $\mathrm{d}-\mathrm{f}$ we present the results for the photoemission intensity $I_{k}(\omega)=A_{k}(\omega) n_{F}(\omega)$, where $A_{k}(\omega)=(1 / \pi) \Sigma_{k}^{\prime \prime}(\omega) /\left(\left(\omega-\epsilon_{k}+\Sigma_{k}^{\prime}(\omega)\right)^{2}+\left(\Sigma^{\prime \prime}(\omega)\right)^{2}\right)$ is the quasiparticle spectral function. Fig. $1 \mathrm{~d}$ shows $I_{k}(\omega)$
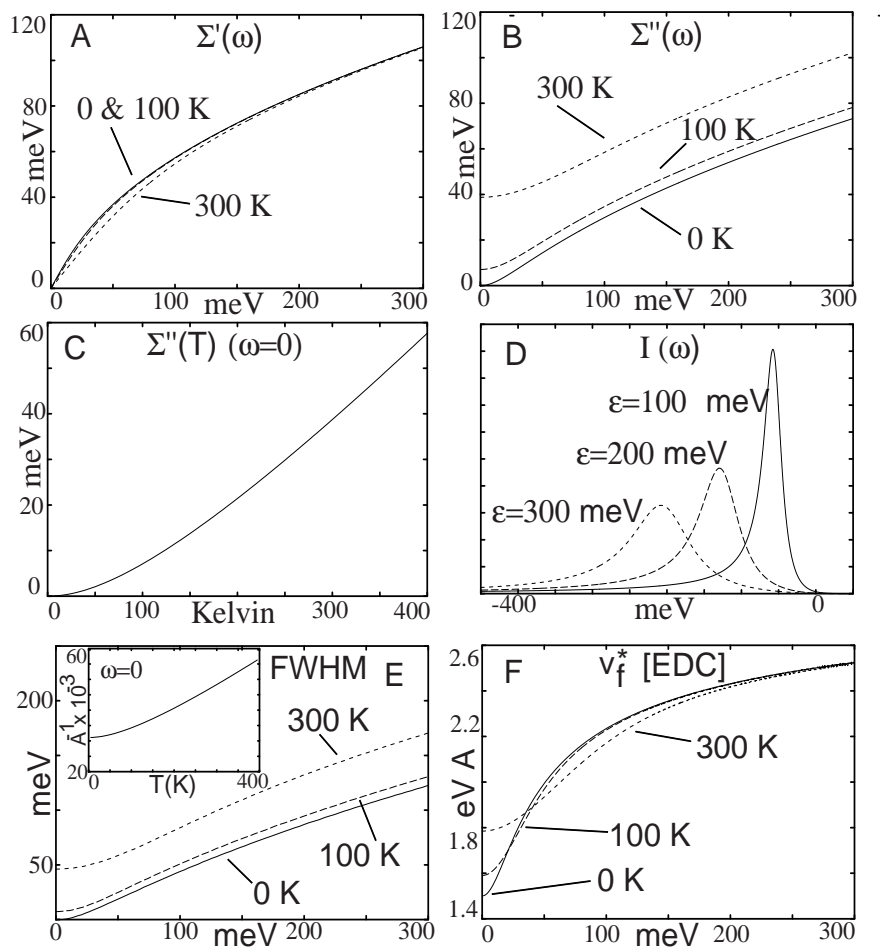

FIG. 1. Theoretical results for the fermionic self-energy, Eq. 1, and the photoemission intensity. For definiteness, we used $\lambda=1, \omega_{s f}=20 \mathrm{meV}$ and $v_{F}=3 \mathrm{eVA}$ (this yields $\xi \approx 9 A)$. Figs. a and $\mathrm{b}-\Sigma^{\prime}(\omega)$ and $\Sigma^{\prime \prime}(\omega)$ respectively at various $T$, indicated on the figures. Fig. $\mathrm{c}-\Sigma^{\prime \prime}(T)$ at $\omega=0$. Fig. d - the photoemission intensity vs $\epsilon_{k}$. Fig. ethe FWHM of the photoemission peak vs $\omega$ at $\epsilon_{k}=0$ (ECD curve). Inset - the FWHM of the peak in $k$-space at $\omega=0$ vs $T$ (MDC curve). Fig. $\mathrm{f}-$ the velocity of the EDC dispersion $v_{F}^{*}=v_{F} /\left(1+d \Sigma^{\prime} / d \omega\right)$ vs $\omega$.

for various $\epsilon_{k}$. Obviously, the width of the peak increases with increasing $\epsilon_{k}$. In Fig.11e, we plot the full width at half maximum (FWHM) of the peak vs $\omega$ at $\epsilon_{k}=0$ (EDC curve). We see that the width scales linearly with frequency over a wide frequency range. In the inset to this figure we plot the FWHM of the peak in $k$-space at $\omega=0$ and varying temperatures (MDC curve). Again, the width clearly scales linearly with $T$ over a substantial $T$-range. In Fig. 1f, we plot the velocity measured in the EDC dispersion $v_{F}^{*}=v_{F} /\left(1+d \Sigma_{k}^{\prime}(\omega) / d \omega\right)$ vs frequency. At $\omega=0, v_{F}^{*}=v_{F} /(1+\lambda)$. At larger frequencies, the renormalization factor decreases due to the flattening of $\Sigma^{\prime}$, and $v_{F}^{*}$ gradually approaches $v_{F}$.

We now discuss how these results are modified away from the hot spots. For this we notice that at finite $\tilde{k}=k-k_{h s}$, Eq. (1) can be reduced to the same form as at a hot spot, if we introduce the $k$ dependent coupling $\lambda_{k}$ and spin fluctuation frequency $\omega_{s f}(k)$ via

$$
\lambda_{k}=\lambda /\left(1+(\tilde{k} \xi)^{2}\right)^{1 / 2}, \quad \omega_{s f}(k)=\omega_{s f}\left(1+(\tilde{k} \xi)^{2}\right)
$$

We see therefore that away from the hot spots, the effective coupling gets smaller, and the crossover fre- 

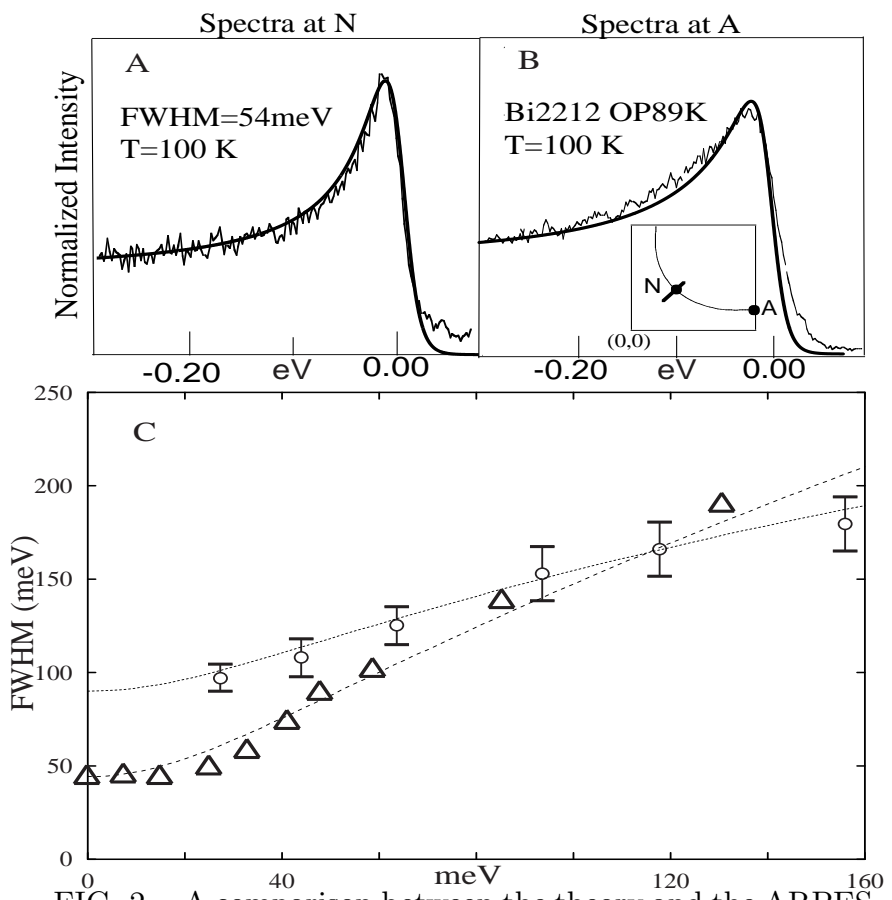

FIG. 2. A comparison between the theory and the ARPES data. a,b. A fit of Argonne data 7 for $k \sim k_{h s}$ (a) and $k=k_{\text {diag }}$ (b); c. Fits of the FWHM of the EDC curves to the theory. The circles with error bars are data from [8] taken at $115 \mathrm{~K}$ and the triangles from 10 taken at $90 \mathrm{~K}$. The two theoretical curves are for different coupling constants (see text)

quency $\omega_{s f}(k)$ increases. Still, however, at frequencies/temperatures which exceed $\omega_{s f}(k), \Sigma_{k}^{\prime \prime}(\omega, T)$ is linear in both frequency and temperature. Obviously then, for intermediate $\omega$, changing the momentum along the Fermi surface only affects the overall slopes of $\Sigma_{k}^{\prime \prime}(\omega)$ vs $\omega$ and $T$. Furthermore, at optimal doing $\xi \sim(1-2) a$, where $a \sim 3 A$ is a $C u-C u$ distance, and $\left|\tilde{k}_{0}\right| \sim 0.3 \pi / a$. Accordingly, $\lambda_{k}$ changes between $\lambda$ and $(0.7-0.4) \lambda$ between $k_{h s}$ and $k_{\text {diag }}$, i.e., the change in the coupling constant is not dramatic.

This analysis is indeed an approximate one, and care must be taken when applying it to momenta about $\mathbf{k}_{F}=\mathbf{k}_{\text {diag }}$ where $\phi_{0}=\pi$, and Eq. (1) is, strictly speaking, unapplicable. This complication certainly affects the fermionic self-energy at the smallest frequencies. However, we verified numerically that for frequencies $\omega \geq \omega_{s f}\left(k_{\text {diag }}\right)$, the dominant contribution to $\Sigma_{k_{\text {diag }}}^{\prime \prime}(\omega)$ comes from fermions away from diagonal, for which $\phi_{0}$ can still be approximated by a constant. We therefoe will use Eq. (1) for momenta both near the hot spots and along the zone diagonals with the understanding that in the latter case, the result has to be modified at the lowest frequencies.

In Fig. 2, we compare our theoretical results with the ARPES data for optimally doped Bi2212. In Figs.2 a and b, we fit ARPES data taken by the Argonne group [7] to our formulas for $k \sim k_{\text {diag }}$ and $k=k_{h s}$. The values $\lambda_{k_{\text {diag }}}=1, \omega_{s f}\left(k_{\text {diag }}\right)=20 \mathrm{meV}$, and $\lambda_{k_{h s}}=2$, $\omega_{s f}\left(k_{h s}\right)=5 \mathrm{meV}$. respectively were used. The value of $\lambda_{k_{h s}}$ agrees with our previous study of the position of the resonance peak at optimal doping [9]. To account for the background, we added a momentum and frequency independent damping $\gamma=70 \mathrm{meV}$ to $\Sigma^{\prime \prime}(\omega)$. This damping likely comes from impurity scattering, but this is not well understood and requires further study.

In Fig. 2fc, we compare the theoretical slope of the FWHM of the EDC curve with the Argonne data for the deviations from the Fermi surface along the zone diagonal. Again we added a constant damping $\gamma=70 \mathrm{meV}$ to $\Sigma^{\prime \prime}(\omega)$. We see that the agreement is quite impressive. The second line in Fig. 2 $c$ is our fit to the Brookhaven 10 and Stanford 11] data, which also yields a linear in frequency FWHM, but with a different slope. To fit this data, we just have to use $\lambda_{k_{d i a g}} \approx 2.5$. This value of the coupling is also quite reasonable. A slightly diffent $\gamma$ (55 meV) was used for the second line. We also verified that the results for the FWHM are largely insensitive to changes in $\omega_{s f}$ which for Fig. 20 is $20 \mathrm{meV}$.

ARPES lineshapes at $k_{h s}$ and $k_{\text {diag }}$ have previously been fitted using the marginal Fermi liquid phenomenology by $\Sigma_{k}^{\prime \prime}(\omega)=A \omega+B_{k}$ with $k$-independent prefactor $A$ 12. Our analysis shows that such data can equally well be fitted by $\Sigma_{k}^{\prime \prime}(\omega)=A_{k} \omega+B$ with a moderately $k$-dependent $A_{k}$ and constant $B$. We emphasize, however, that the theoretical reasoning is qualitatively different in the two approaches.

We now turn to the computation of the conductivity $\sigma(\omega)=i \Pi(\omega) / \omega$, where $\Pi(\omega)$ is a current-current correlator. Diagramatically, $\Pi(\omega)$ is given by a particle-hole bubble with $d \epsilon_{k} / d k$ in the vertices. In our calculations, the typical momenta are comparable to $k_{F}$, and hence vertices can be treated as constants. Also, since the selfenergy, Eq. (1), depends predominantly on frequency, vertex corrections to the particle-hole bubble (related to $d \Sigma / d k$ by the Ward identity) do not change the physics and can be safely neglected. For $k$ near the hot spots, the neglect of vertex corrections can also be justified by the argument that the velocities at $k$ and $k+Q$ are nearly orthogonal, and hence the transport lifetime is the same as the conventional lifetime.

Substituting the expressions for the self-energy into the particle-hole bubble and expanding, as before, to linear order in deviations from the Fermi surface, one can explicitly perform the integration over $\epsilon_{k}$ and obtain

$\Pi\left(\omega_{m}\right)=\frac{\omega_{p l}^{2}}{4} T \sum_{n} \int d \tilde{k} \frac{\Theta\left(\omega_{n}+\omega_{m}\right)-\Theta\left(\omega_{n}\right)}{\omega_{m}+\Sigma_{k}\left(\omega_{n}+\omega_{m}\right)-\Sigma_{k}\left(\omega_{n}\right)}$

where the momentum integration is along the Fermi surface, $\Sigma_{k}\left(\omega_{n}\right)$ is given by (1), $\Theta(x)$ is a Theta-function, and $\omega_{p l}$ is the plasma frequency. We used $\omega_{p l} \sim 1.2 \times$ $10^{4} \mathrm{~cm}^{-1}$, similar to that in 13 . 

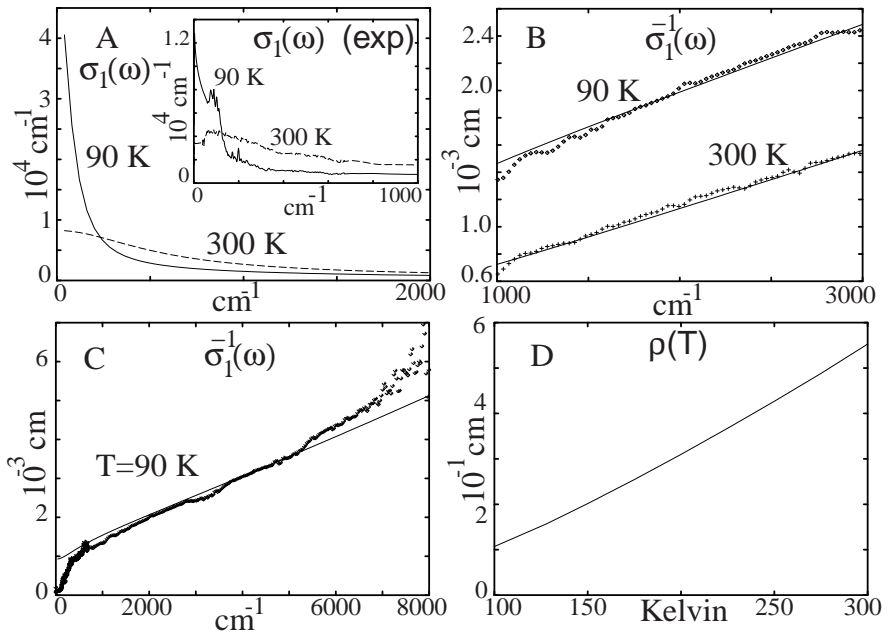

FIG. 3. Optical conductivity at optimal doping. For definiteness we used $\lambda=2, \omega_{s f}=5 \mathrm{meV}$ a. The theoretical results for $\sigma_{1}(\omega)$. The experimental data is from 13 is shown in the inset; b. $\sigma_{1}^{-1}$ vs $\omega$ for $T=90 K$ and $T=300 K$. The lines are theoretical curves, and the points are the experimental data; c. $\sigma_{1}^{-1}(\omega)$ in the extended frequency range. The experimental data is from Ref [13]; e)the resistivity $\rho(T)$ vs $T$.

The $k$-dependence of $\Sigma_{k}(\omega)$ emerges through the momentum dependence of $\lambda_{k}$ and $\omega_{s f}(k)$. As this dependence is not dramatic at optimal doping, it should not substantially affect the frequency dependence of $\Pi(\omega)$. To make the computations more transparent, we replace $\lambda_{k}$ and $\omega_{s f}(k)$ by averaged, $k$-independent values.

We computed $\Pi^{\prime \prime}(\omega)$ by using a spectral representation, and used a Kramers-Kronig transformation to obtain $\Pi^{\prime}(\omega)$. In Fig. 3 a we present our theoretical results for $\sigma_{1}(\omega)=\operatorname{Re} \sigma(\omega)$ at $\mathrm{T}=90$ and $300 \mathrm{~K}$. The inset of this figure contains experimental data from Ref [13]. In Fig. $3 \mathrm{~b}$, we plot $1 / \sigma_{1}(\omega)$ for frequencies above $1000 \mathrm{~cm}^{-1}$ and compare it with the data. We see that the theoretical $1 / \sigma_{1}(\omega)$ is linear in frequency. This fully agrees with the data. Furthermore, we found that $\lambda=2$, compatible to that which we used for the spectral function, yields a perfect fit to the measured slopes of $1 / \sigma_{1}(\omega)$ both at $90 K$ and $300 K$. We consider this agreement a strong indication that spin-fluctuation mechanism captures the essential physics of the cuprates. To match the values of the conductivity, we, however, again have to add a frequency independent (but temperature dependent) constant to $1 / \sigma_{1}$. As roughly, $1 / \sigma_{1}(\omega) \propto \Sigma^{\prime \prime}(\omega)$, this procedure is qualitatively similar (although not quite equivalent) to adding a constant to $\Sigma^{\prime \prime}(\omega)$. Similar procedure has been used in Ref. [3]. In Fig. 3c we plot our $1 / \sigma_{1}(\omega)$ in the extended frequency range, up to $8000 \mathrm{~cm}^{-1}$. We see that the theoretical linear behavior of $1 / \sigma_{1}$ extends up to very large frequencies of $7000 \mathrm{~cm}^{-1}$, where $\Sigma^{\prime \prime}(\omega)$ already curves down from a linear behavior. This extension of the linear regime to very high frequencies is also consistent with the experimental data of 13. Finally, in
Fig. 3e, we present the result for the resistivity $\rho(T)$. We see that $\rho(T)$ is linear in $T$ above $100 K$. This is indeed a consequence of the linearity of $\Sigma^{\prime \prime}(T, \omega=0)$.

To summarize, in this paper we used the spinfluctuation approach to calculate the photoemission intensity, optical conductivity and resistivity in the normal state of the cuprates near optimal doping. We found that the fermionic self-energy is linear in both $\omega$ and $T$ in a wide range of frequencies and temperatures. This gives rise to a linear frequency dependence of the inverse conductivity and to a linear temperature dependence of the resistivity. We performed quantitative comparisons with the experimental data and found near perfect matches of the slopes for both the ARPES linewidth and the conductivity. We view the results as a strong indication that the interaction between low-energy fermions and their spin collective degrees of freedom is the dominant scattering mechanism in the cuprates. The open issue is whether the spin-fluctuation scenario is capable of explaining the differences between the temperature dependence of the diagonal and Hall conductivities [3,14,15]. This study is currently under way.

It is our pleasure to thank D. Basov, G. Blumberg, J.C. Campuzano P. Johnson, M. Norman, J. Schmalian, O. Tschernishev and V. Yakovenko for useful discussions. We are also thankful to D. Basov for sending us the unpublished experimental data. The research was supported by NSF DMR-9979749 (Ar. A and A. Ch.) and by NSF DMR-96-32527 (R.H.).

[1] P.W. Anderson, The Theory of Superconductivity in the High $T_{c}$ Cuprates (Princeton University Press, Princeton, New Jersey, 1997).

[2] C. Varma et al., Phys. Rev. Lett. 63, 1996 (1989), ibid 64, 497 (1990). C.M. Varma, Phys. Rev. B 55, 14554 (1997). A different but somewhat similar in spirit is the scenario based on the assumed closeness to a charge density wave instability: C. Castellani, C. DiCastro and M. Grilli, Phys. Rev. Lett. 75, 4650 (1995).

[3] L. B. Ioffe and A. J. Millis, Phys. Rev. B, 58, 11631 (1998).

[4] R. Hlubina and T.M. Rice, Phys. Rev. B, 52, 13043 (1995).

[5] A. Chubukov, Europhys. Lett. 44, 655 (1997); Ar. Abanov and A. Chubukov, Phys. Rev. Lett., 83, 1652 (1999). Ar. Abanov, A. Chubukov, and A. Finkel'stein, cond-mat/9911445.

[6] T.P. Devereaux and A.P. Kampf, Int. J. Mod. Phys. B 11, 2093 (1997).

[7] A. Kaminski et al., Phys. Rev. Lett. 84, 1788 (2000).

[8] A. Kaminski et al., cond-mat/0004482.

[9] Ar. Abanov, A. Chubukov, and J. Schmalian, condmat/0005163. 
[10] T. Valla et al., Science, 285, 2210 (1999).

[11] Z-X Shen et al., Science, 280, 259 (1998).

[12] E. Abrahams and C. Varma, Proc. Natl. Acad. of Sciences 97, 5714 (2000).

[13] A. Puchkov, D. Basov and T. Timusk, J. Phys.: Cond. Matter 8, 10049 (1996). D. Basov, private communication.

[14] B.P. Stojkovic and D. Pines, Phys. Rev. B 55, 8576 (1997); ibid 56, 11931 (1997); see also A. Rosch, condmat/9910432.

[15] A. T. Zheleznyak, V. M. Yakovenko, and H. D. Drew, Phys. Rev. B 59, 207 (1999). 